\title{
Unidirectional laser emission from $\pi$-conjugated polymer microcavities with broken symmetry
}

A. Tulek, and Z. V. Vardeny

Citation: Appl. Phys. Lett. 90, 161106 (2007); doi: 10.1063/1.2723078

View online: http://dx.doi.org/10.1063/1.2723078

View Table of Contents: http://aip.scitation.org/toc/apl/90/16

Published by the American Institute of Physics

\section{Articles you may be interested in}

Whispering-gallery mode microdisk lasers

Applied Physics Letters 60, 289 (1998); 10.1063/1.106688

Vertical microgoblet resonator with high sensitivity fabricated by direct laser writing on a Si substrate Journal of Applied Physics 121, 064502 (2017); 10.1063/1.4975790

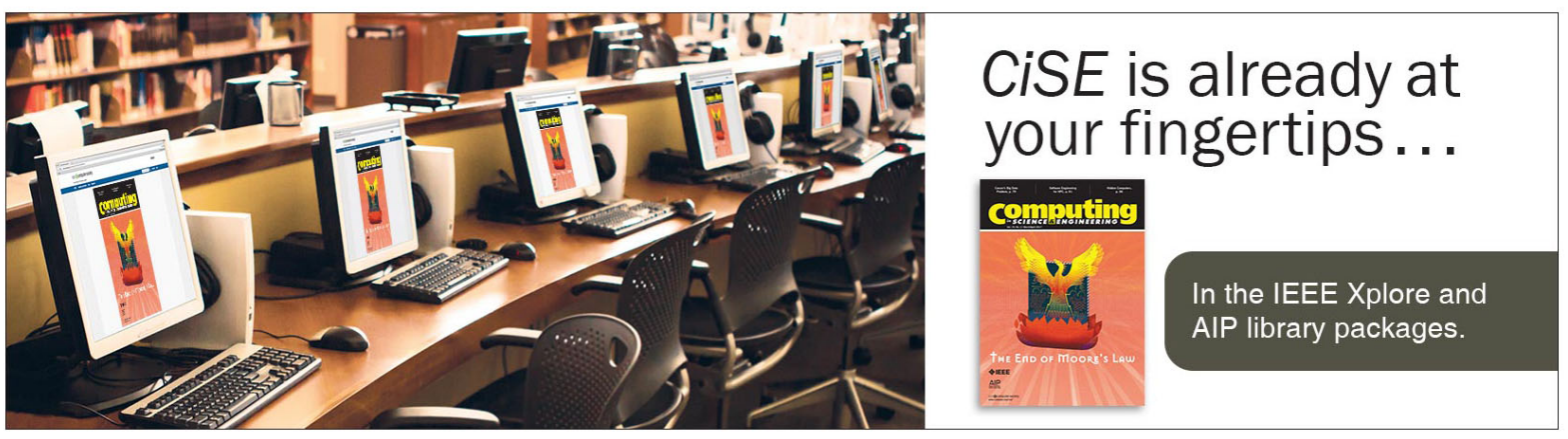




\title{
Unidirectional laser emission from $\pi$-conjugated polymer microcavities with broken symmetry
}

\author{
A. Tulek ${ }^{\mathrm{a})}$ and Z. V. Vardeny ${ }^{\mathrm{b})}$ \\ Department of Physics, University of Utah, Salt Lake City, Utah 84112
}

(Received 24 December 2006; accepted 15 March 2007; published online 16 April 2007)

\begin{abstract}
We report unidirectional laser emission from $\pi$-conjugated polymer microcavities with broken symmetry geometries such as spiral and microdisk containing a "line defect," in comparison with plain microdisk cavity having isotropic emission. We found that the laser emission directionality contrast ratio is $8-10$ and far field lateral divergence angle is $12^{\circ}-15^{\circ}$ for both broken symmetry geometries, with no significant increase in the laser threshold intensity. Fourier transform analysis of the laser emission spectra shows that unlike microdisks with line defect, the variation of light trajectories in the spiral microcavities leads to less defined laser modes. () 2007 American Institute of Physics. [DOI: 10.1063/1.2723078]
\end{abstract}

Laser emission from microresonators that support whispering gallery modes (WGMs) such as microdisk, microring, and microsphere has been extensively studied in recent years because of the ease of fabrication and lower threshold intensity due to very high quality factors of such resonators. ${ }^{1-5}$ There exists, however, an important drawback of these highly symmetric microcavities, namely, lack of emission directionality: a feature that is inferior in the field of laser action. Different approaches have been employed to overcome this negative by deforming the circular shape into quadruple, ${ }^{6}$ and ellipse, ${ }^{7}$ for example. In addition, a variety of other geometrical structures such as stadium shape ${ }^{8}$ have been fabricated for improving the emission directionality. However, higher laser threshold is required for obtaining laser emission from such irregular microcavities, and, in addition these microcavities also produce multiple output beams, thus splitting the emission energy flux.

Therefore additional efforts have been devoted to outcoupling the trapped light inside the microresonator without substantially deforming its symmetry. For example, spiral microcavities formed using both inorganic and organic semiconductors were recently shown to have directional emission with relatively high quality factor. ${ }^{9-12}$ However, only a broad lateral divergence angle of $\sim 70^{\circ}$ was demonstrated for the organic microspiral lasers. ${ }^{11}$ Very recently another microcavity with broken symmetry was theoretically introduced, ${ }^{13}$ which is based on a relatively long "line defect" in a plain microdisk that is formed away from the circumference. In this case the WGM field at the circumference "tunnels" toward the defect line, which then assumes a role of a secondary light source of which emission is enhanced in a direction perpendicular to the line defect. ${ }^{13}$ However, so far no experimental verification of such laser microcavity has been reported. In the present work we study unidirectional laser emission from $\pi$-conjugated polymer microcavities with broken symmetry geometries such as spiral, and microdisk with line defect, and compare their laser emission properties to those of a plain microdisk.

\footnotetext{
a) Present address: Department of Physics, Bilkent University, 06800 Bilkent, Ankara, Turkey.

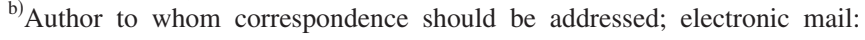
val@physics.utah.edu
}

For the organic semiconductor gain material we used poly(dioctyloxy) phenyl vinylene (DOO-PPV), a red-emitter $\pi$-conjugated polymer with superior emission quantum efficiency ${ }^{14}$ [see Fig. 1(a) inset]. The polymer powder was dissolved in toluene and spin cast onto a glass substrate forming a uniform film with thickness of $\sim 2 \mu \mathrm{m}$. A positive photoresist was spun onto the polymer film and "soft baked"

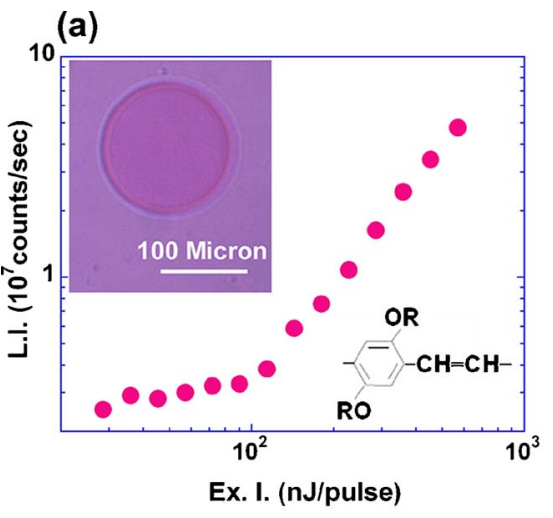

(b)

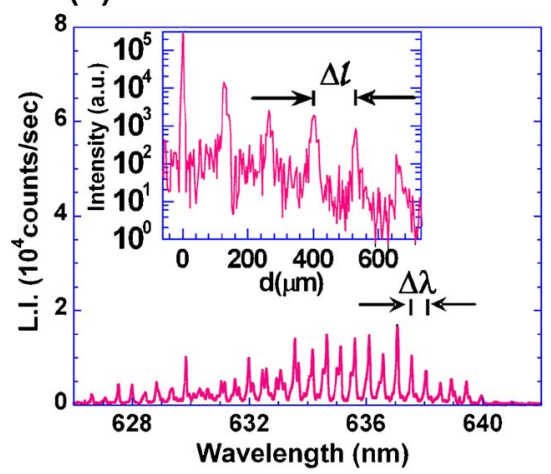

FIG. 1. (Color online) Plain microdisk cavity: (a) The emission intensity vs excitation energy showing laser threshold $I_{\text {th }} \sim 90 \mathrm{~nJ} /$ pulse. The insets are the optical images of the $155 \mu \mathrm{m}$ diameter microdisk and chemical structure of the DOO-PPV polymer. The abbreviations L.I. and Ex. I. stand for light intensity and excitation intensity, respectively; $d$ is the PFT parameter that is correlated to the wavelength (in $\mathrm{cm}^{-1}$ ). (b) The laser emission spectrum for $I>I(\max )$; the inset shows the PFT of the emission spectrum, where $d$ is the Fourier transform parameter that is correlated with the emission wavelength. $\Delta \lambda$ in the emission spectrum and $\Delta l$ in the PFT are assigned. 
(a)

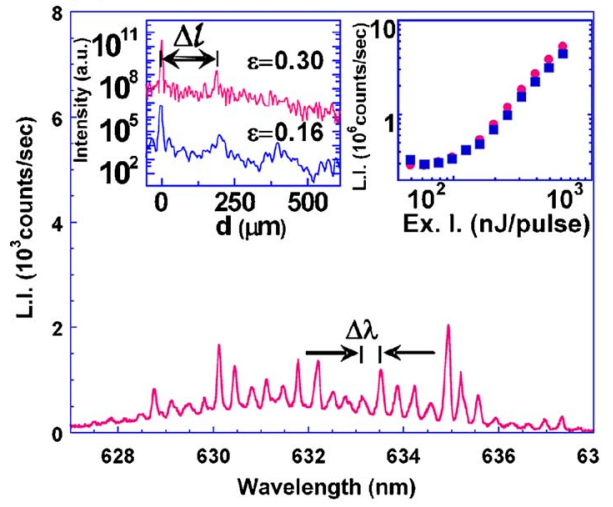

(b)

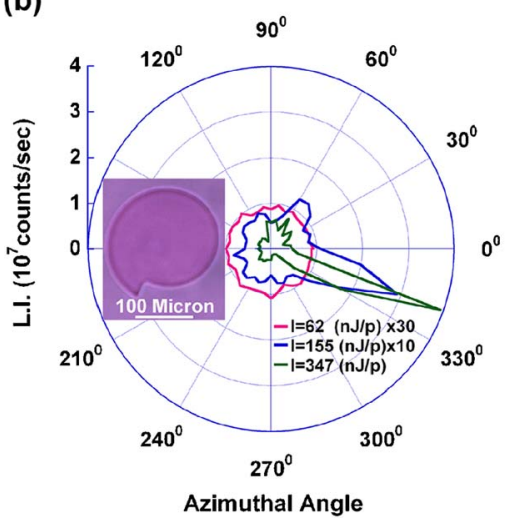

FIG. 2. (Color online) Microspiral cavity: (a) The laser emission spectrum and its PFT (left inset) of two microspirals with (i) $\varepsilon=0.30$ (red/gray line) and (ii) $\varepsilon=0.16$ (blue/full line). The right inset is the laser emission vs $I$, showing $I_{\text {th }} \sim 100 \mathrm{~nJ} /$ pulse for both microcavities. L.I., Ex. I., and $d$ are the same as in Fig. 1. (b) The azimuthal emission intensity distribution for microspiral (i) at $I<I_{\text {th }}$ (red/gray line) and $I>I_{\text {th }}$ [green and blue (full) lines]. The inset is the optical image of the microspiral cavity (i) with $r_{0}=79 \mu \mathrm{m}$.

at $90{ }^{\circ} \mathrm{C}$. After the UV exposure and developing, the structures that were preprinted on the photoresist were "hard baked" onto the film at $120^{\circ} \mathrm{C}$, and etched with oxygen plasma onto the polymer layer. The microcavity diameters ranged from 50 to $200 \mu \mathrm{m}$ with a boundary roughness $<1 \mu \mathrm{m}$ [Fig. 1(a) inset]. Spiral microcavities were fabricated, where the radius varies according to the relation $r(\phi)=r_{0}(1+\varepsilon \phi / 2 \pi),{ }^{10}$ where $r_{0}$ is the smallest radius and $\varepsilon$ is the deformation factor [Fig. 2(a) inset]. For our microcavities $r_{0}$ ranged from 50 to $150 \mu \mathrm{m}$, and $\varepsilon$ ranged from 0.15 to 0.30 . We fabricated line defects in plain polymer microdisks having a fixed width of $2 \mu \mathrm{m}$ and a length of 10 or $20 \mu \mathrm{m}$. The distance from the defect edge to the microdisk circumference was $\sim 10 \mu \mathrm{m}$. The orientation of the line defect with respect to the circular geometry [Fig. 3(a) inset] that is characterized by an angle $\theta$ [Fig. 3(b) inset], was set to $\theta=45^{\circ}$, $60^{\circ}$, or $75^{\circ}$.

Laser emission from the fabricated microcavities was generated using the second harmonic of a pulsed Nd:yttriumaluminum-garnet laser amplifier system operating at $532 \mathrm{~nm}$, with pulse duration of $100 \mathrm{ps}$ at $870 \mathrm{~Hz}$ repetition rate. The emitted light was collected using a fiber of $1 \mathrm{~mm}$ diameter, approximately $1 \mathrm{~cm}$ away from microcavities and sent to a triple spectrometer, where a charged coupled device camera recorded the light intensity as photon counts. The overall spectral resolution of the collected light emission was $0.1 \mathrm{~nm}$. A manual rotational stage having $2^{\circ}$ accuracy was
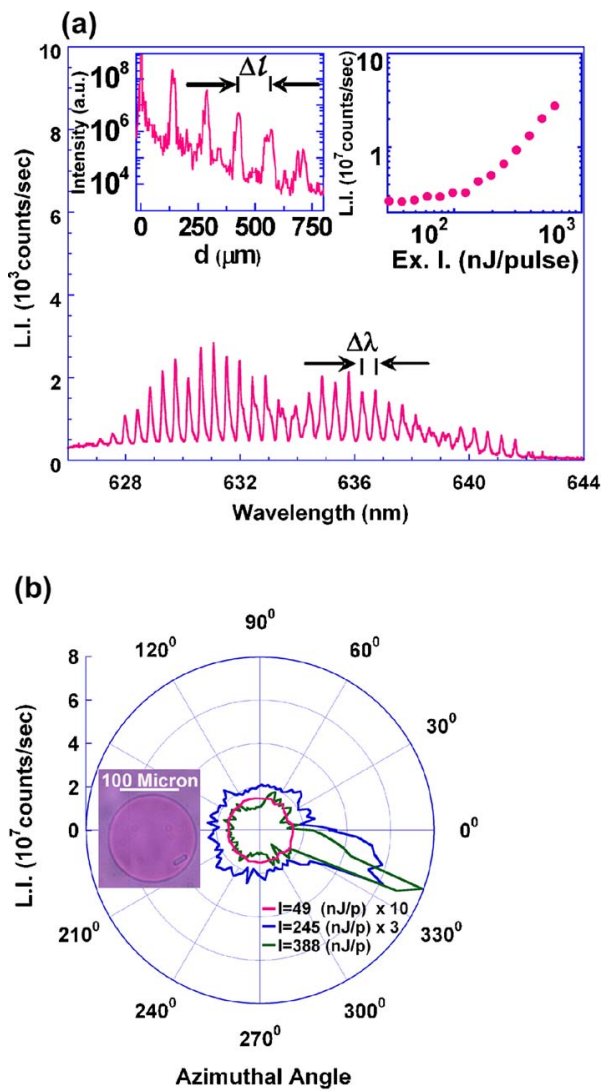

FIG. 3. (Color online) Microdisk cavity with line defect: (a) The laser emission spectrum and its PFT (left inset). The right inset shows the emission intensity vs $I$ measured at an azimuthal angle of $340^{\circ}$ showing $I_{\text {th }} \sim 100 \mathrm{~nJ} /$ pulse. (b) The azimuthal emission intensity distribution for the line defect microdisk cavity at $I<I_{\text {th }}$ (red/gray line) and $I>I_{\text {th }}$ [green and blue (full) lines]. The inset is the microcavity optical image, where $D$ $=155 \mu \mathrm{m}$ and the line defect size is $2 \times 20 \mu \mathrm{m}^{2}$ with $\theta=60^{\circ}$.

used to rotate the laser microstructures for azimuthal intensity distribution measurements, and the recorded intensity was plotted versus the azimuthal angle $\varphi$.

The spectral laser mode spacing $\Delta \lambda$ in the emission of such microcavities above laser threshold is given by the relation ${ }^{14} \Delta \lambda=\lambda^{2} / n C$, where $\lambda$ is the emission wavelength, $n$ is the polymer effective refractive index, and $C$ is the microresonator circumference. For microdisk with fixed diameter $D, C^{\prime}=\pi D$, whereas for spiral microcavity $C^{\star}$ was calculated by integrating the arc length for a complete cycle in $\phi$. In addition, the power Fourier transform (PFT) of the emission spectrum from a laser microcavity contains equally spaced diminishing Fourier components with periodicity $\Delta l=n C / 2 \pi .^{14}$

The following laser characteristic properties for a plain microdisk serve as a basis comparison for the two broken symmetry microlasers discussed later. The bilinear logarithmic dependence of the emission output intensity on the excitation intensity $I$ presented in Fig. 1(a) for a plain microdisk with $D=155 \mu \mathrm{m}$ shows a laser threshold $I_{\text {th }} \sim 90 \mathrm{~nJ} /$ pulse. A number of narrow emission lines at $\sim 634 \mathrm{~nm}$ are observed with equidistant mode spacing, $\Delta \lambda$ $\sim 0.44 \mathrm{~nm}$ [Fig. 1(b)], which gives rise to a PFT spectrum that contains periodic peaks separated by $\Delta l=n D / 2$ $=138 \mu \mathrm{m}$. From this relation we calculate the polymer effective refractive index upon laser action to be $n \sim 1.78$. In addition, we found that the azimuthal distribution of the emission intensity for excitation intensities below and above laser 
threshold is isotropic (within 5\%), as expected for this type of symmetric microcavity.

From the emission versus excitation intensity dependence shown in Fig. 2(a) right inset the threshold intensity $I_{\text {th }}$ of two spiral cavities with $r_{0}=79 \mu \mathrm{m}$ and $\varepsilon=0.30$, and $r_{0}=86 \mu \mathrm{m}$ and $\varepsilon=0.16$, respectively, was measured to be $\sim 100 \mathrm{~nJ} /$ pulse; comparable to that of the corresponding microdisk. The laser emission spectrum of the former spiral microcavity [Fig. 2(a)] contains similar modes to that of the plain microdisk, but they do not possess a well-defined mode separation. This is clearly seen in the PFT analysis of the emission spectrum, which contains only a single harmonic peak [Fig. 2(a) left inset]. This finding can be explained when considering the variation of the light trajectories in a spiral geometry, which produces a bigger variation in the effective $n C^{\star}$ value. ${ }^{9,12}$ Yet the variation of such trajectories is reduced at smaller $\varepsilon$ approaching the microdisk case, as shown in Fig. 2(a) left inset for $\varepsilon=0.16$. Nevertheless from the relation $\Delta l=n C^{\star} / 2 \pi$, where $n=1.78$ as determined above, we found that the effective circumference of the spiral cavity for $\varepsilon=0.30$ is $646 \pm 4 \mu \mathrm{m}$. The physical circumference of this cavity is calculated to be $\sim 650 \mu \mathrm{m}$, which is in excellent agreement with the PFT analysis. This microcavity clearly shows unidirectional emission [Fig. 2(b)]. The emission from the spiral microcavity with $\varepsilon=0.30$ has a narrow lateral divergence angle (LDA) $\sim 12^{\circ}$. It also has a directionality contrast ratio (DCR), which is defined as the ratio between maximum and minimum emission intensities along the azimuthal angle, of $\sim 10$, as measured at tilt angle $\alpha \sim 20^{\circ}$. These directionality parameters are better than those achieved previously with another polymer lasing medium. ${ }^{11}$ We also note that the DCR improves at higher excitation intensities and/or larger cavity deformation factor.

As seen in Fig. 3(a) right inset we also measured $I_{\text {th }} \sim 100 \mathrm{~nJ} /$ pulse independent of the fabricated line defect parameters, similar to that of the perfect microdisk. In addition the laser emission spectrum preserves its ordered line structure due to the disk shape [Fig. 3(a)]. This indicates that the WGM field in such cavities is not disturbed much by the introduced "linear defect," since is relatively far from the circumference; but, in contrast close enough that the WGM field tunnels through and creates a secondary light source with less divergence. ${ }^{13}$ From the line separation in the emission PFT [Fig. 3(a)] we obtained an effective diameter $D=151 \pm 4 \mu \mathrm{m}$, in very good agreement with the original fabricated diameter of $\sim 155 \mu \mathrm{m}$. Importantly, unidirectional emission was observed from such microstructure with DCR of $\sim 8$ and LDA of $\sim 15^{\circ}$ [Fig. 3(b)]. No considerable difference in directionality has been observed with respect to the size or orientation of the introduced line defect, in contrast with the theory. ${ }^{13}$ This may be explained by the experimental variation in disk diameter and film thickness, which obscure the predicted changes in the DCR with the linear defect parameters. We note that the laser mode linewidth for this type of cavity is the same as in plain microdisk, and this shows that the microlaser quality factor is determined by impurities, defects, self absorption, and inhomogeneity in the polymer film, rather than by the cavity quality factor.

In conclusion, we fabricated microcavities with broken symmetry from DOO-PPV luminescent polymer films and observed very promising unidirectional laser emission with directionality contrast ratio of $8-10$ and lateral divergence angle of $12^{\circ}-15^{\circ}$. The improved emission directionality does not come at the expense of $I_{\mathrm{th}}$, and this indicates that the microlaser quality factor for polymer lasers is determined by factors other than the resonator quality factor. This shows that directional emission in polymer lasers can be obtained without substantial increase in the excitation intensity. The microdisk cavity containing line defect has an advantage over the spiral microcavity, since it preserves the equidistant mode spacing in the laser emission spectrum along with similar improved directionality properties of the emission radiation.

The authors thank M. Raikh for many useful discussions and R. Polson for help with the measurements. This work was supported in part by the DOE Grant No. 04-ER 46109 and the NSF DMR Grant No. 05-03172.

${ }^{1}$ S. L. McCall, A. F. J. Levi, R. E. Slusher, S. J. Pearton, and R. A. Logan, Appl. Phys. Lett. 60, 289 (1991).

${ }^{2}$ A. F. J. Levi, R. E. Slusher, S. L. McCall, S. J. Pearton, and W. S. Hobson, Appl. Phys. Lett. 62, 2021 (1993).

${ }^{3}$ M. K. Gonokami, R. H. Jordan, A. Dodabalapur, H. E. Katz, M. L. Schilling, R. E. Slusher, and S. Ozawa, Opt. Lett. 20, 2093 (1995).

${ }^{4}$ V. Sandoghdar, F. Treussart, J. Hare, V. L. Seguin, J. M. Raimond, and S. Haroche, Phys. Rev. A 54, R1777 (1996).

${ }^{5}$ R. C. Polson, Z. V. Vardeny, and D. A. Chinn, Appl. Phys. Lett. 81, 1561 (2002).

${ }^{6}$ J. U. Nockel, A. D. Stone, G. Chen, H. L. Grossman, and R. K. Chang, Opt. Lett. 21, 1609 (1996).

${ }^{7}$ B. Gayral, J. M. Gerard, B. Legrand, E. Costard, and V. T. Mieg, Appl. Phys. Lett. 72, 1421 (1998).

${ }^{8}$ C. Gmachl, F. Capasso, E. E. Narimanov, J. U. Nockel, A. D. Stone, J. F. Deborah, L. Sivco, and A. Y. Cho, Science 280, 1556 (1998).

${ }^{9}$ G. D. Chern, H. E. Tureci, A. D. Stone, R. K. Chang, M. Kneissl, and N. M. Johnson, Appl. Phys. Lett. 83, 1710 (2003).

${ }^{10}$ M. Kneissl, M. Teepe, N. Miyashita, N. M. Johnson, G. D. Chern, and R. K. Chang, Appl. Phys. Lett. 84, 2485 (2004).

${ }^{11}$ A. Fujii, T. Nishimura, Y. Yoshida, K. Yoshino, and M. Ozaki, Jpn. J. Appl. Phys., Part 2 44, L1091 (2005).

${ }^{12}$ F. Courvoisier, V. Boutou, J. P. Wolf, R. K. Chang, and J. Zyss, Opt. Lett. 30, 738 (2005).

${ }^{13}$ V. M. Apalkov and M. E. Raikh, Phys. Rev. B 70, 195317 (2004).

${ }^{14}$ R. C. Polson, G. Levina, and Z. V. Vardeny, Appl. Phys. Lett. 76, 3858 (2000). 